\title{
Article \\ Event-Triggered Impulsive Optimal Control for Continuous-Time Dynamic Systems with Input Time-Delay
}

\author{
Quanyu Bai and Wei Zhu * (D)
}

check for updates

Citation: Bai, Q.; Zhu, W. Event-Triggered Impulsive Optimal Control for Continuous-Time

Dynamic Systems with Input Time-Delay. Mathematics 2022, 10, 279. https://doi.org/10.3390/math 10020279

Academic Editor: Jen-Chih Yao

Received: 12 December 2021

Accepted: 14 January 2022

Published: 17 January 2022

Publisher's Note: MDPI stays neutral with regard to jurisdictional claims in published maps and institutional affiliations.

Copyright: (C) 2022 by the authors. Licensee MDPI, Basel, Switzerland. This article is an open access article distributed under the terms and conditions of the Creative Commons Attribution (CC BY) license (https:// creativecommons.org/licenses/by/ $4.0 /)$.
Key Lab of Intelligent Analysis and Decision on Complex Systems, Chongqing University of Posts and Telecommunications, Chongqing 400065, China; s190603003@stu.cqupt.edu.cn

* Correspondence: zhuwei@cqupt.edu.cn

\begin{abstract}
Time-delay is an inevitable factor in practice, which may affect the performance of optimal control. In this paper, the event-triggered impulsive optimal control for linear continuous-time dynamic systems is studied. The event-triggered impulsive optimal feedback controller with input time-delay is presented, where the impulsive instants are determined by some designed eventtriggering function and condition depending on the state of the system. Some sufficient conditions are given for guaranteeing the exponential stability with the optimal controller. Moreover, the Zenobehavior for the impulsive instants is excluded. Finally, an example with numerical simulation is given to verify the validity of the theoretical results.
\end{abstract}

Keywords: event-triggered impulsive control; optimal control; zeno-behavior; time-delay

\section{Introduction}

Optimal control is an important branch of modern control theory. The system can realize the optimization of the performance index during the process from the initial state to the terminal state under the optimal control. By using the optimal control theory, the purpose of energy and time minimization can be achieved, which plays an important role in realizing the efficient utilization of resources, reducing energy consumption and improving economic benefits. Optimal control theory has important applications in robotics [1], vehicles [2], aerospace [3,4], medical and chemical industry [5], and so on.

Time-delay is ubiquitous in many practical systems. It comes from different sources, such as measurement delays, transportation delays and communication lags. Under the influence of time-delay, system performance will be deteriorated, and even the system will become unstable [6,7]. Thus, it is necessary to study the optimal control of systems with time-delay. In [8], an optimal feedback control based on integral reinforcement learning was proposed for linear continuous systems with input time-delay. In $[9,10]$, optimal regulators for linear systems with single and multiple input time-delay was investigated, respectively. In [11], the time-delay stochastic optimal control problem with nonconvex control domain and diffusion term containing both control and its delayed term was investigated. In [12], the optimal control for mean-field continuous-time systems subject to input time-delay was studied. The optimal control for linear system with input time-delay was studied, this delay system was transformed into a delay-free linear system, and a new optimal feedback controller was designed in [13].

In addition, impulsive effect widely exists in dynamic systems. One of the advantages of the impulse effect is that it can stabilize an unstable system by using a few small pulses, which widely applied in practice, such as ecosystem management [14], treatment and transmission of epidemic diseases [15,16], orbit transfer of communications satellites [17], etc. Meanwhile, impulsive optimal control problems have been extensively concerned. However, it should be pointed out that the impulsive instants in [18-20] were fixed and predetermined or just the upper and lower bounds of impulsive intervals were given. This 
sampling method, independent of the state of the system, may lead to high frequency of controller update, and result in the waste of computing resources. To address these issue, Event-triggered Control (ETC) method was proposed in [21,22]. Then, combining ETC and impulsive control, more and more researchers have developed the event-triggered impulsive control (ETIC), where the event-triggering functions and conditions are pre-set, and impulsive instants are determined by some specific events [23,24]. ETIC is expected to reduce communication load and controller updates frequency, which motivates its wide applications. In [25], the exponential stability of nonlinear systems with input time-delay by means of ETIC was studied. In [26], ETIC approach was proposed to achieve synchronization of leader-following coupled dynamical systems. In [27], the synchronization problem of neural network was studied by using ETIC, the static and dynamic event-triggering conditions were proposed, respectively. Under the ETIC strategy, stabilization of impulsive systems with stochastic time-delay was discussed in [28]. Although these results with ETIC have been investigated, the problem of ETIC of systems with input time-delay, even for linear case, is seldom considered. Hence, establishing optimal control results for linear systems via ETIC, especially for systems with input time-delay, is still a challenge.

Based on the above discussions, the event-triggered impulsive optimal control for linear continuous-time dynamic system is investigated in this paper. In Section 2, considering the influence of input time-delay, an optimal state feedback controller with time delay is designed, where the impulsive instants are determined by some designed event-triggering functions and conditions, which depend on the state of the system. In Section 3, based on stability theory, some sufficient conditions are given for guaranteeing the exponential stability with the optimal controller. Moreover, the Zeno-behavior for the impulsive instants is excluded. In Section 4, the validity of the theoretical results is verified by using an example with numerical simulation. Conclusion and future study are made in Section 5.

Notations: $R^{m \times n}$ denotes the set of matrix with $m$ rows and $n$ columns, and all the elements are real numbers. For $x \in R^{n}, A \in R^{n \times n},\|x\|$ and $\|A\|$ are the Euclidean norm and the corresponding induced matrix norm, respectively.

\section{Preliminaries}

Consider the following linear continuous-time dynamic system

$$
\dot{x}=A x(t)+B u(t-\tau),
$$

where $A \in R^{n \times n}, B \in R^{n \times m}$, and $x(t) \in R^{n}$ assumed completely measurable is the system state, $u(t-\tau) \in R^{m \times 1}$ is the control variable, $\tau$ is the input time-delay. We assume that $(\mathrm{A}, \mathrm{B})$ is stabilizable and the control occurs when $t \geq \tau$.

Based on the advantage of impulsive control, the following impulsive control will be used

$$
\left\{\begin{array}{cl}
u(t-\tau) & =K x\left(t_{k}-\tau\right), \quad \text { for } t \in\left[t_{k}, t_{k+1}\right), \\
x(t) & =C_{k} x\left(t^{-}\right), \quad \text { for } t=t_{k}, k=1,2, \cdots,
\end{array}\right.
$$

where $x\left(t^{-}\right)$denotes the left limitation of $x(t), K \in R^{m \times n}$ is the control gain matrix to be designed later.

Impulsive control for linear continuous-time dynamic systems has attracted extensive research $[13,29,30]$. However, the impulsive instants in $[13,29,30]$ were given in advance, which were independent of the system, and may cause unnecessary update of controller.

In this paper, event-triggered mechanism is used to determine the impulsive instants dynamically. The event-triggering impulsive instants $t_{k}(k=1,2, \cdots)$ are defined iteratively by [24]

$$
t_{k+1}=\inf \left\{t \in R \mid t>t_{k}, f(t) \geq 0\right\},
$$

where

$$
f(t)=\|e(t)\|-\sigma\left\|x\left(t_{k}\right)\right\|-\beta e^{-\lambda\left(t-t_{0}\right)}
$$


is said to be the event-triggering function for some $\sigma \in(0,1), \beta>0, \lambda>0$ and $e(t)=$ $x\left(t_{k}\right)-x(t) . e(t)$ is reset to 0 at each impulsive instant. By (1) and (2), we can obtain

$$
\left\{\begin{array}{l}
\dot{x}(t)=A x(t)+B K(x(t-\tau)+e(t-\tau)), \text { for } t \in\left[t_{k}, t_{k+1}\right), \\
x(t)=C_{k} x\left(t^{-}\right), \quad \text { for } t=t_{k}, k=1,2, \cdots .
\end{array}\right.
$$

The problem of this paper is to design $K$ and $C_{k}$, such that the following integral quadratic cost function $J$ reaches a minimum value [31]

$$
\begin{aligned}
J= & \frac{1}{2} \int_{0}^{\infty}\left[x^{T}(t) Q x(t)+u^{T}(t) W u(t)\right] d t \\
& +\frac{1}{2} \sum_{k=1}^{\infty} x^{T}\left(t_{k}^{-}\right) Q_{k} x\left(t_{k}^{-}\right),
\end{aligned}
$$

where $Q=Q^{T} \geq 0, Q_{k}=Q_{k}^{T} \geq 0$ and $W=W^{T}>0$ are of appropriate dimensions.

Based on Lemma 2.1 in [13] and the necessary conditions for optimality in [31], with mild revision, we have the following lemma.

Lemma 1. Consider the liner continuous-time dynamic system (1). Let $K=-W^{-1} B^{T} P$. Then, the optimal control can be expressed in the following form

$$
u^{*}(t-\tau)=-W^{-1} B^{T} P x\left(t_{k}-\tau\right),
$$

where $P \in R^{n \times n}$ is the symmetric positive definite solution of the Algebraic Riccati Equation (ARE) for any positive semidefinite matrix $Q$

$$
P A+A^{T} P-P B_{0} W^{-1} B_{0}^{T} P+Q=0,
$$

where $B_{0}=e^{-A \tau} B$.

Remark 1. $Q_{k}$ in the cost function (6) can be determined by the following formula

$$
\left(e^{A \tau}\right)^{T} P e^{A \tau}=Q_{k}+C_{k}^{T}\left(e^{A \tau}\right)^{T} P e^{A \tau} C_{k},
$$

with some given $C_{k}$.

Definition 1 ([25]). Given a locally Lipschitz function $V: R^{n} \rightarrow R_{+}$, the upper right-hand Dini derivative of $V$ along system (1) is defined by

$$
D^{+} V[f]=\lim _{h \rightarrow 0^{+}} \sup \frac{1}{h} V(x+h f)-V(x) .
$$

Definition 2 ([32]). System (1) is said to achieve event-triggered impulsive optimal control with exponential convergence rate, if there are positive constants $M>0$ and $\alpha>0$ such that the solution of the system (5) satisfies

$$
\|x(t)\|=M\left\|x\left(t_{0}\right)\right\| \exp \left(-\alpha\left(t-t_{0}\right)\right), t \geq t_{0} .
$$

Definition 3 ([32]). There is no Zeno-behavior for the impulsive instants if inf $\left\{t_{k+1}-t_{k}\right\}>0$.

\section{Main Results}

Inspired by the demonstrations in $[13,31,33,34]$, we get the following two theorems. 


\subsection{Stability Analysis}

In this section, exponential stability of system (1) with the proposed optimal controller is discussed. Denote $G=A^{T}+A+2 B K K^{T} B^{T}, a_{1}=1+\frac{\sigma^{2}}{(1-\sigma)^{2}}, a_{2}=\frac{2 \sigma \beta}{(1-\sigma)^{2}}, a_{3}=\frac{\beta^{2}}{(1-\sigma)^{2}}$, $\sup _{k}\left\{t_{k+1}-t_{k}\right\} \leq \rho<\infty$.

Theorem 1. Consider the liner continuous-time dynamic system (1) with $K=-W^{-1} B^{T} P$ and the impulsive instants (3). If there are impulsive gain matrices $C_{k}$ satisfying $\left\|C_{k}\right\|<\gamma<1$ and $a_{1} e^{2 \lambda \tau}+\gamma\left(2 \lambda+\lambda_{\max }(G)+\frac{\ln \gamma}{\rho}\right)<0$. Then, the event-triggered impulsive optimal control can be achieved with exponential convergence rate for any $\tau \geq 0, \sigma \in(0,1)$.

Proof. Let $V(t)=x^{T}(t) x(t)$ be the Lyapunov candidate function. For $t \in\left[t_{k}, t_{k+1}\right)$, computing the time derivative of $V(t)$ along the trajectory of (5) yields

$$
\begin{aligned}
\dot{V}(t)= & \dot{x}^{T}(t) x(t)+x^{T}(t) \dot{x}(t) \\
= & \left(A x(t)+B K x\left(t_{k}-\tau\right)\right)^{T} x(t) \\
& +x^{T}(t)\left(A x(t)+B K x\left(t_{k}-\tau\right)\right) \\
= & x^{T}(t)\left(A^{T}+A\right) x(t)+x^{T}\left(t_{k}-\tau\right) K^{T} B^{T} x(t) \\
& +x^{T}(t) B K x\left(t_{k}-\tau\right) \\
= & x^{T}(t)\left(A^{T}+A\right) x(t)+x^{T}(t-\tau) K^{T} B^{T} x(t) \\
& +e^{T}(t-\tau) K^{T} B^{T} x(t)+x^{T}(t) B K x(t-\tau) \\
& +x^{T}(t) B K e(t-\tau) \\
= & x^{T}(t)\left(A^{T}+A\right) x(t)+2 x^{T}(t) B K x(t-\tau) \\
& +2 x^{T}(t) B K e(t-\tau) \\
\leq & x^{T}(t)\left(A^{T}+A\right) x(t) \\
& +2 x^{T}(t) B K K^{T} B^{T} x(t) \\
& +x^{T}(t-\tau) x(t-\tau)+e^{T}(t-\tau) e(t-\tau) \\
\leq & x^{T}(t)\left(A^{T}+A+2 B K K^{T} B^{T}\right) x(t) \\
& +x^{T}(t-\tau) x(t-\tau)+e^{T}(t-\tau) e(t-\tau) .
\end{aligned}
$$

By event-triggering condition in (3), it holds

$$
\|e(t)\| \leq \frac{\sigma\|x(t)\|+\beta e^{-\lambda\left(t-t_{0}\right)}}{1-\sigma} .
$$

Then,

$$
\begin{aligned}
\dot{V}(t) \leq & \lambda_{\max }(G)\|x(t)\|^{2}+\|x(t-\tau)\|^{2}+\|e(t-\tau)\|^{2} \\
\leq & \lambda_{\max }(G)\|x(t)\|^{2}+\|x(t-\tau)\|^{2}+\left(\frac{\sigma\|x(t-\tau)\|+\beta e^{-\lambda\left(t-\tau-t_{0}\right)}}{1-\sigma}\right)^{2} \\
\leq & \lambda_{\max }(G)\|x(t)\|^{2}+\|x(t-\tau)\|^{2} \\
& +\frac{\sigma^{2}}{(1-\sigma)^{2}}\|x(t-\tau)\|^{2}+\frac{2 \sigma \beta e^{-\lambda\left(t-\tau-t_{0}\right)}}{(1-\sigma)^{2}}\|x(t-\tau)\|+\frac{\beta^{2} e^{-2 \lambda\left(t-\tau-t_{0}\right)}}{(1-\sigma)^{2}} \\
\leq & \lambda_{\max }(G) V(t)+\left(1+\frac{\sigma^{2}}{(1-\sigma)^{2}}\right)\|x(t-\tau)\|^{2} \\
& +\frac{2 \sigma \beta e^{-\lambda\left(t-\tau-t_{0}\right)}}{(1-\sigma)^{2}}\|x(t-\tau)\|+\frac{\beta^{2} e^{-2 \lambda\left(t-\tau-t_{0}\right)}}{(1-\sigma)^{2}} .
\end{aligned}
$$


By the variation of parameter formula, one can derive that

$$
\begin{aligned}
V(t) \leq & e^{\lambda_{\max }(G)\left(t-t_{k}\right)} V\left(t_{k}\right) \\
& +\int_{t_{k}}^{t} e^{\lambda_{\max }(G)(t-s)}\left(\left(1+\frac{\sigma^{2}}{(1-\sigma)^{2}}\right)\|x(s-\tau)\|^{2}+\right. \\
& \left.\frac{2 \sigma \beta e^{-\lambda\left(s-\tau-t_{0}\right)}}{(1-\sigma)^{2}}\|x(s-\tau)\|+\frac{\beta^{2} e^{-2 \lambda\left(s-\tau-t_{0}\right)}}{(1-\sigma)^{2}}\right) d s .
\end{aligned}
$$

It follows that

$$
\begin{aligned}
\|x(t)\|^{2} \leq & e^{\lambda_{\max }(G)\left(t-t_{k}\right)}\left\|x\left(t_{k}\right)\right\|^{2} \\
& +\int_{t_{k}}^{t} e^{\lambda_{\max }(G)(t-s)}\left(\left(1+\frac{\sigma^{2}}{(1-\sigma)^{2}}\right)\|x(s-\tau)\|^{2}+\right. \\
& \left.\frac{2 \sigma \beta e^{-\lambda\left(s-\tau-t_{0}\right)}}{(1-\sigma)^{2}}\|x(s-\tau)\|+\frac{\beta^{2} e^{-2 \lambda\left(s-\tau-t_{0}\right)}}{(1-\sigma)^{2}}\right) d s .
\end{aligned}
$$

At impulsive instants $t_{k}, k=1,2, \cdots$, there exist appropriate matrices $\left\|C_{k}\right\| \leq \gamma<1$, $k=1,2, \cdots$. It follows from the discrete part of $(2),(5)$ that

$$
\begin{aligned}
\left\|x\left(t_{k}\right)\right\|^{2} & \leq\left\|C_{k}\right\|^{2} \cdot\left\|x\left(t_{k}^{-}\right)\right\|^{2} \\
& \leq\left\|C_{k}\right\|\left\|x\left(t_{k}^{-}\right)\right\|^{2} \\
& \leq \gamma\left\|x\left(t_{k}^{-}\right)\right\|^{2}, k=1,2, \cdots .
\end{aligned}
$$

Then, for $\mathrm{t} \in\left[t_{1}, t_{2}\right)$, we have

$$
\begin{aligned}
\|x(t)\|^{2} \leq & e^{\lambda_{\max }(G)\left(t-t_{1}\right)}\left\|x\left(t_{1}\right)\right\|^{2} \\
+ & \int_{t_{1}}^{t} e^{\lambda_{\max }(G)(t-s)}\left(\left(1+\frac{\sigma^{2}}{(1-\sigma)^{2}}\right)\|x(s-\tau)\|^{2}+\right. \\
& \left.\frac{2 \sigma \beta e^{-\lambda\left(s-\tau-t_{0}\right)}}{(1-\sigma)^{2}}\|x(s-\tau)\|+\frac{\beta^{2} e^{-2 \lambda\left(s-\tau-t_{0}\right)}}{(1-\sigma)^{2}}\right) d s,
\end{aligned}
$$

and

$$
\begin{aligned}
\left\|x\left(t_{1}\right)\right\|^{2} \leq & \gamma\left\|x\left(t_{1}^{-}\right)\right\|^{2} \\
= & \gamma\left\{e^{\lambda_{\max }(G)\left(t_{1}-t_{0}\right)}\left\|x\left(t_{0}\right)\right\|^{2}\right. \\
& +\int_{t_{0}}^{t_{1}} e^{\lambda_{\max }(G)\left(t_{1}-s\right)}\left(\left(1+\frac{\sigma^{2}}{(1-\sigma)^{2}}\right)\|x(s-\tau)\|^{2}+\right. \\
& \left.\left.\frac{2 \sigma \beta e^{-\lambda\left(s-\tau-t_{0}\right)}}{(1-\sigma)^{2}}\|x(s-\tau)\|+\frac{\beta^{2} e^{-2 \lambda\left(s-\tau-t_{0}\right)}}{(1-\sigma)^{2}}\right) d s\right\} .
\end{aligned}
$$


Thus, for $t \in\left[t_{1}, t_{2}\right)$, we obtain that

$$
\begin{aligned}
\|x(t)\|^{2} \leq & \gamma e^{\lambda_{\max }(G)\left(t-t_{0}\right)}\left\|x\left(t_{0}\right)\right\|^{2} \\
+ & \gamma \int_{t_{0}}^{t_{1}} e^{\lambda_{\max }(G)(t-s)}\left(\left(1+\frac{\sigma^{2}}{(1-\sigma)^{2}}\right)\|x(s-\tau)\|^{2}\right. \\
& \left.\frac{2 \sigma \beta e^{-\lambda\left(s-\tau-t_{0}\right)}}{(1-\sigma)^{2}}\|x(s-\tau)\|+\frac{\beta^{2} e^{-2 \lambda\left(s-\tau-t_{0}\right)}}{(1-\sigma)^{2}}\right) d s \\
+ & \int_{t_{1}}^{t} e^{\lambda_{\max }(G)(t-s)}\left(\left(1+\frac{\sigma^{2}}{(1-\sigma)^{2}}\right)\|x(s-\tau)\|^{2}+\right. \\
& \left.\frac{2 \sigma \beta e^{-\lambda\left(s-\tau-t_{0}\right)}}{(1-\sigma)^{2}}\|x(s-\tau)\|+\frac{\beta^{2} e^{-2 \lambda\left(s-\tau-t_{0}\right)}}{(1-\sigma)^{2}}\right) d s .
\end{aligned}
$$
holds

In generally, by mathematical induction, for $t \in\left[t_{k}, t_{k+1}\right)$, the following inequality

$$
\begin{aligned}
\|x(t)\|^{2} \leq & \prod_{t_{0}<t_{k}<t} \gamma e^{\lambda_{\max }(G)\left(t-t_{0}\right)}\left\|x\left(t_{0}\right)\right\|^{2} \\
+ & \int_{t_{0}}^{t} \prod_{s<t_{k}<t} \gamma e^{\lambda_{\max }(G)(t-s)}\left(\left(1+\frac{\sigma^{2}}{(1-\sigma)^{2}}\right)\|x(s-\tau)\|^{2}+\right. \\
& \left.\frac{2 \sigma \beta e^{-\lambda\left(s-\tau-t_{0}\right)}}{(1-\sigma)^{2}}\|x(s-\tau)\|+\frac{\beta^{2} e^{-2 \lambda\left(s-\tau-t_{0}\right)}}{(1-\sigma)^{2}}\right) d s
\end{aligned}
$$

where $\prod_{s<t_{k}<t} \gamma=\gamma^{l}, l$ is the total number of impulsive instant $t_{k}$ satisfying $t_{k} \in(s, t)$.

In term of (12), we have

$$
\begin{aligned}
\|x(t)\|^{2} \leq & \gamma^{\frac{t-t_{0}}{\rho}-1} e^{\lambda_{\max }(G)\left(t-t_{0}\right)}\left\|x\left(t_{0}\right)\right\|^{2} \\
& +\int_{t_{0}}^{t} \gamma^{\frac{t-s}{\rho}-1} e^{\lambda_{\max }(G)(t-s)}\left(\left(1+\frac{\sigma^{2}}{(1-\sigma)^{2}}\right)\|x(s-\tau)\|^{2}+\right. \\
& \left.\frac{2 \sigma \beta e^{-\lambda\left(s-\tau-t_{0}\right)}}{(1-\sigma)^{2}}\|x(s-\tau)\|+\frac{\beta^{2} e^{-2 \lambda\left(s-\tau-t_{0}\right)}}{(1-\sigma)^{2}}\right) d s \\
\leq & \frac{1}{\gamma} e^{\left(\lambda_{\max }(G)+\frac{\ln \gamma}{\rho}\right)\left(t-t_{0}\right)}\left\|x\left(t_{0}\right)\right\|^{2} \\
& +\int_{t_{0}}^{t} \frac{1}{\gamma} e^{\left(\lambda_{\max }(G)+\frac{\ln \gamma}{\rho}\right)(t-s)}\left(\left(1+\frac{\sigma^{2}}{(1-\sigma)^{2}}\right)\|x(s-\tau)\|^{2}+\right. \\
& \left.\frac{2 \sigma \beta e^{-\lambda\left(s-\tau-t_{0}\right)}}{(1-\sigma)^{2}}\|x(s-\tau)\|+\frac{\beta^{2} e^{-2 \lambda\left(s-\tau-t_{0}\right)}}{(1-\sigma)^{2}}\right) d s .
\end{aligned}
$$

In the following, for any $\eta>1$, it will be proved that

$$
\|x(t)\|<\eta Z e^{-\lambda\left(t-t_{0}\right)}=\|v(t)\|, t \geq t_{0},
$$

where $Z=\max \left\{\frac{1}{\sqrt{\gamma}}\left\|x\left(t_{0}\right)\right\|, \frac{-a_{2} e^{2 \lambda \tau}-\sqrt{a_{2}^{2} e^{4 \lambda \tau}-4\left(a_{1} e^{2 \lambda \tau}+\gamma\left(2 \lambda+\lambda_{\max }(G)+\frac{\ln \gamma}{\rho}\right)\right) a_{3} e^{2 \lambda \tau}}}{2\left(a_{1} e^{2 \lambda \tau}+\gamma\left(2 \lambda+\lambda_{\max }(G)+\frac{\ln \gamma}{\rho}\right)\right)}\right\}$.

Otherwise, there must exist $a t^{*}>t_{0}$ such that

$$
\left\|x\left(t^{*}\right)\right\|^{2}=\left\|v\left(t^{*}\right)\right\|^{2} \text { and }\|x(t)\|<\|v(t)\|, \text { for } t \in\left[t_{0}, t^{*}\right) .
$$


By (13), we can get that

$$
\begin{aligned}
& \left\|v\left(t^{*}\right)\right\|^{2}=\left\|x\left(t^{*}\right)\right\|^{2} \\
& \leq \frac{1}{\gamma} e^{\left(\lambda_{\max }(G)+\frac{\ln \gamma}{\rho}\right)\left(t^{*}-t_{0}\right)}\left\|x\left(t_{0}\right)\right\|^{2} \\
& +\int_{t_{0}}^{t^{*}} \frac{1}{\gamma} e^{\left(\lambda_{\max }(G)+\frac{\ln \gamma}{\rho}\right)\left(t^{*}-s\right)}\left[a_{1}\|x(s-\tau)\|^{2}\right. \\
& \left.+a_{2} e^{-\lambda\left(s-\tau-t_{0}\right)}\|x(s-\tau)\|+a_{3} e^{-2 \lambda\left(s-\tau-t_{0}\right)}\right] d s \\
& <\frac{\eta^{2}}{\gamma} e^{\left(\lambda_{\max }(G)+\frac{\ln \gamma}{\rho}\right)\left(t^{*}-t_{0}\right)}\left\|x\left(t_{0}\right)\right\|^{2} \\
& +\int_{t_{0}}^{t^{*}} \frac{1}{\gamma} e^{\left(\lambda_{\max }(G)+\frac{\ln \gamma}{\rho}\right)\left(t^{*}-s\right)}\left[a_{1} \eta^{2} Z^{2} e^{-2 \lambda\left(s-\tau-t_{0}\right)}\right. \\
& \left.+a_{2} \eta^{2} Z e^{-2 \lambda\left(s-\tau-t_{0}\right)}+a_{3} \eta^{2} e^{-2 \lambda\left(s-\tau-t_{0}\right)}\right] d s \\
& =\frac{\eta^{2}}{\gamma} e^{\left(\lambda_{\max }(G)+\frac{\ln \gamma}{\rho}\right)\left(t^{*}-t_{0}\right)}\left\|x\left(t_{0}\right)\right\|^{2} \\
& +\int_{t_{0}}^{t^{*}} \frac{a_{1} \eta^{2} Z^{2}+a_{2} \eta^{2} Z+a_{3} \eta^{2}}{\gamma} e^{2 \lambda \tau} e^{\left(\lambda_{\max }(G)+\frac{\ln \gamma}{\rho}\right)\left(t^{*}-s\right)} e^{-2 \lambda\left(s-t_{0}\right)} d s \\
& =\frac{\eta^{2}}{\gamma} e^{\left(\lambda_{\max }(G)+\frac{\ln \gamma}{\rho}\right)\left(t^{*}-t_{0}\right)}\left\|x\left(t_{0}\right)\right\|^{2} \\
& -\frac{\left(a_{1} \eta^{2} Z^{2}+a_{2} \eta^{2} Z+a_{3} \eta^{2}\right) e^{2 \lambda \tau}}{\gamma\left(2 \lambda+\lambda_{\max }(G)+\frac{\ln \gamma}{\rho}\right)}\left[e^{-2 \lambda\left(t^{*}-t_{0}\right)}-e^{\left(\lambda_{\max }(G)+\frac{\ln \gamma}{\rho}\right)\left(t^{*}-t_{0}\right)}\right] \\
& =\frac{\eta^{2}}{\gamma}\left\{e^{\left(\lambda_{\max }(G)+\frac{\ln \gamma}{\rho}\right)\left(t^{*}-t_{0}\right)}\left\|x\left(t_{0}\right)\right\|^{2}\right. \\
& \left.-\frac{a_{1} Z^{2}+a_{2} Z+a_{3}}{2 \lambda+\lambda_{\max }(G)+\frac{\ln \gamma}{\rho}} e^{2 \lambda \tau}\left[e^{-2 \lambda\left(t^{*}-t_{0}\right)}-e^{\left(\lambda_{\max }(G)+\frac{\ln \gamma}{\rho}\right)\left(t^{*}-t_{0}\right)}\right]\right\} .
\end{aligned}
$$

Case I: $\mathrm{Z}=\frac{1}{\sqrt{\gamma}}\left\|x\left(t_{0}\right)\right\|$, which implies that

$$
\frac{1}{\gamma}\left\|x\left(t_{0}\right)\right\|^{2}+\frac{a_{1} Z^{2}+a_{2} Z+a_{3}}{\gamma\left(2 \lambda+\lambda_{\max }(G)+\frac{\ln \gamma}{\rho}\right)} e^{2 \lambda \tau} \geq 0,
$$

then, by (15), we obtain that

$$
\left\|v\left(t^{*}\right)\right\|^{2}<\frac{\eta^{2}}{\gamma} e^{-2 \lambda\left(t^{*}-t_{0}\right)}\left\|x\left(t_{0}\right)\right\|^{2}=\eta^{2} Z^{2} e^{-2 \lambda\left(t^{*}-t_{0}\right)}=\left\|v\left(t^{*}\right)\right\|^{2} .
$$

Case II: $Z=\frac{-a_{2} e^{2 \lambda \tau}-\sqrt{a_{2}^{2} e^{4 \lambda \tau}-4\left(a_{1} e^{2 \lambda \tau}+\gamma\left(2 \lambda+\lambda_{\max }(G)+\frac{\ln \gamma}{\rho}\right)\right) a_{3} e^{2 \lambda \tau}}}{2\left(a_{1} e^{2 \lambda \tau}+\gamma\left(2 \lambda+\lambda_{\max }(G)+\frac{\ln \gamma}{\rho}\right)\right)}$, which implies that

$$
\frac{1}{\gamma}\left\|x\left(t_{0}\right)\right\|^{2}+\frac{a_{1} Z^{2}+a_{2} Z+a_{3}}{\gamma\left(2 \lambda+\lambda_{\max }(G)+\frac{\ln \gamma}{\rho}\right)} e^{2 \lambda \tau}<0,
$$

then, by (15), we obtain that

$$
\begin{aligned}
\left\|v\left(t^{*}\right)\right\|^{2} & <-\eta^{2} \frac{a_{1} Z^{2}+a_{2} Z+a_{3}}{\gamma\left(2 \lambda+\lambda_{\max }(G)+\frac{\ln \gamma}{\rho}\right)} e^{2 \lambda \tau} e^{-2 \lambda\left(t^{*}-t_{0}\right)} \\
& =\eta^{2} Z^{2} e^{-2 \lambda\left(t^{*}-t_{0}\right)}=\left\|v\left(t^{*}\right)\right\|^{2}
\end{aligned}
$$

The contradiction in (16) or (17) reveals that (14) is valid for any $\eta>1$. Let $\eta \rightarrow 1$, one has

$$
\|x(t)\| \leq Z e^{-\lambda\left(t-t_{0}\right)}, t \geq t_{0}
$$

which implies event-triggered impulsive optimal control is achieved with exponential convergence rate. 


\subsection{Exclusion of the Zeno-Behavior}

Theorem 2. Zeno-behavior for impulsive instants determined by (3) is excluded with the same conditions as Theorem 1.

Proof. Computing the right-upper Dini derivative of $\|e(t)\|$ along the solution of (5) over interval $\left[t_{k}, t_{k+1}\right)$, we have

$$
\begin{aligned}
D^{+}\|e(t)\| \leq & \|\dot{e}(t)\|=\|\dot{x}(t)\| \\
= & \|A x(t)+B K(x(t-\tau)+e(t-\tau))\| \\
\leq & \|A\|\|x(t)\|+\|B K\|\|x(t-\tau)\|+\|B K\|\|e(t-\tau)\| \\
\leq & \|A\|\|x(t)\|+\|B K\|\|x(t-\tau)\| \\
& +\sigma\|B K\|\left\|x\left(t_{k}-\tau\right)\right\|+\|B K\| \beta e^{-\lambda\left(t-\tau-t_{0}\right)} \\
\leq & \|A\| Z e^{-\lambda\left(t-t_{0}\right)}+\|B K\| Z e^{-\lambda\left(t-\tau-t_{0}\right)} \\
& +\sigma Z\|B K\| e^{-\lambda\left(t_{k}-\tau-t_{0}\right)}+\|B K\| \beta e^{-\lambda\left(t-\tau-t_{0}\right)} .
\end{aligned}
$$

Since $e\left(t_{k}\right)=0$, it follows from (19) that

$$
\begin{aligned}
\|e(t)\| \leq & \int_{t_{k}}^{t}\left[\|A\| Z e^{-\lambda\left(s-t_{0}\right)}+\|B K\| Z e^{-\lambda\left(s-\tau-t_{0}\right)}\right. \\
& \left.+\sigma Z\|B K\| e^{-\lambda\left(t_{k}-\tau-t_{0}\right)}+\|B K\| \beta e^{-\lambda\left(s-\tau-t_{0}\right)}\right] d s \\
\leq & \frac{\|A\| Z}{\lambda}\left[e^{-\lambda\left(t_{k}-t_{0}\right)}-e^{-\lambda\left(t-t_{0}\right)}\right] \\
& +\frac{\|B K\| Z}{\lambda}\left[e^{-\lambda\left(t_{k}-\tau-t_{0}\right)}-e^{-\lambda\left(t-\tau-t_{0}\right)}\right] \\
& +\frac{\|B K\| \beta}{\lambda}\left[e^{-\lambda\left(t_{k}-\tau-t_{0}\right)}-e^{-\lambda\left(t-\tau-t_{0}\right)}\right] \\
& +\sigma Z\|B K\| e^{-\lambda\left(t_{k}-\tau-t_{0}\right)}\left(t-t_{k}\right) .
\end{aligned}
$$

The next event will not be triggered until triggering function crosses zero, i.e.,

$$
\begin{aligned}
\sigma\left\|x\left(t_{k+1}\right)\right\|+\beta e^{-\lambda\left(t_{k+1}-t_{0}\right)} \leq & \left\|e\left(t_{k+1}\right)\right\| \\
\leq & \frac{\|A\| Z}{\lambda}\left[e^{-\lambda\left(t_{k}-t_{0}\right)}-e^{-\lambda\left(t_{k+1}-t_{0}\right)}\right] \\
& +\frac{\|B K\| Z}{\lambda}\left[e^{-\lambda\left(t_{k}-\tau-t_{0}\right)}-e^{-\lambda\left(t_{k+1}-\tau-t_{0}\right)}\right] \\
& +\frac{\|B K\| \beta}{\lambda}\left[e^{-\lambda\left(t_{k}-\tau-t_{0}\right)}-e^{-\lambda\left(t_{k+1}-\tau-t_{0}\right)}\right] \\
& +\sigma Z\|B K\| e^{-\lambda\left(t_{k}-\tau-t_{0}\right)}\left(t_{k+1}-t_{k}\right) .
\end{aligned}
$$

Denote $T_{k}=t_{k+1}-t_{k}$

$$
\begin{aligned}
\beta e^{-\lambda T_{k}} \leq & \frac{\|A\| Z}{\lambda}\left[1-e^{-\lambda T_{k}}\right]+\frac{\|B K\| Z}{\lambda} e^{\lambda \tau}\left[1-e^{-\lambda T_{k}}\right] \\
& +\frac{\|B K\| \beta}{\lambda} e^{\lambda \tau}\left[1-e^{-\lambda T_{k}}\right]+\sigma Z\|B K\| e^{\lambda \tau} T_{k} .
\end{aligned}
$$

By (21), one can easily derive that

$$
\begin{array}{r}
\left\{T_{k}>0: \frac{\|A\| Z}{\lambda}\left[1-e^{-\lambda T_{k}}\right]+\frac{\|B K\| Z}{\lambda} e^{\lambda \tau}\left[1-e^{-\lambda T_{k}}\right]\right. \\
\left.+\frac{\|B K\| \beta}{\lambda} e^{\lambda \tau}\left[1-e^{-\lambda T_{k}}\right]+\sigma Z\|B K\| e^{\lambda \tau} T_{k}-\beta e^{-\lambda T_{k}} \geq 0\right\}
\end{array}
$$

is nonempty and $T=\inf \left\{T_{k}\right\}>0$, which implies that the Zeno-behavior is excluded. 


\section{Numerical Simulations}

In this section, an example is presented to illustrate the theoretical results obtained in Section 3. Consider linear continuous-time dynamic system (1) with

$$
A=\left(\begin{array}{ccc}
-0.46 & -0.26 & 0.04 \\
-0.21 & 0.11 & 0.16 \\
-0.04 & -0.64 & -0.14
\end{array}\right), B=\left(\begin{array}{l}
1 \\
0 \\
0
\end{array}\right)
$$

In order to obtain the optimal control gain matrix $\mathrm{K}$, parameter matrices $\mathrm{Q}$ and $\mathrm{W}$ are chosen as $Q=\left(\begin{array}{lll}1 & 0 & 0 \\ 0 & 1 & 0 \\ 0 & 0 & 1\end{array}\right), \mathrm{W}=10$.

Then, $\mathrm{P}$ can be obtained by (8) and then

$$
K=-W^{-1} B^{T} P=\left(\begin{array}{lll}
-0.2014 & 0.8468 & 0.0858
\end{array}\right) .
$$

The impulsive gain matrices $C_{k}$ are designed as $C_{k}=\left(\begin{array}{ccc}0.002 & 0 & -0.048 \\ -0.08 & -0.01 & -0.01 \\ -0.01 & -0.01 & 0.022\end{array}\right)$, $k=1,2, \cdots,\left\|C_{k}\right\|=0.0818$.

Then, we have $Q_{k}=\left(\begin{array}{ccc}7.23 & 7.29 & 7.28 \\ 7.29 & 7.32 & 7.32 \\ 7.28 & 7.32 & 7.32\end{array}\right)$.

Let $\left\|C_{k}\right\|<\gamma<1, \rho=0.11$.

Therefore, for any bounded $\tau \geq 0, \sigma \in(0,1), \beta>0, \lambda>0$, the event-triggered impulsive optimal control is achieved with exponential convergence rate by Theorem 1 .

In term of Algorithm 1, let the initial condition be $x_{0}(t)=\left[\begin{array}{lll}-0.9 & 0.5 & 0.8\end{array}\right]^{\mathrm{T}}, t \in\left[\begin{array}{l}-\tau, 0\end{array}\right]$. If $\tau=0.1, \gamma=0.253, \sigma=0.22, \lambda=0.05, \beta=0.1$. Then, $a_{1} e^{2 \lambda \tau}+\gamma\left(2 \lambda+\lambda_{\max }(G)+\right.$ $\left.\frac{\ln \gamma}{\rho}\right)=-0.0085<0$. If $\tau=1, \gamma=0.28, \sigma=0.22, \lambda=0.05, \beta=0.1$. Then, $a_{1} e^{2 \lambda \tau}+\gamma\left(2 \lambda+\lambda_{\max }(G)+\frac{\ln \gamma}{\rho}\right)=-0.0076<0$. The simulation results are shown in Figures 1-8, where the impulsive instants are described by $*$.

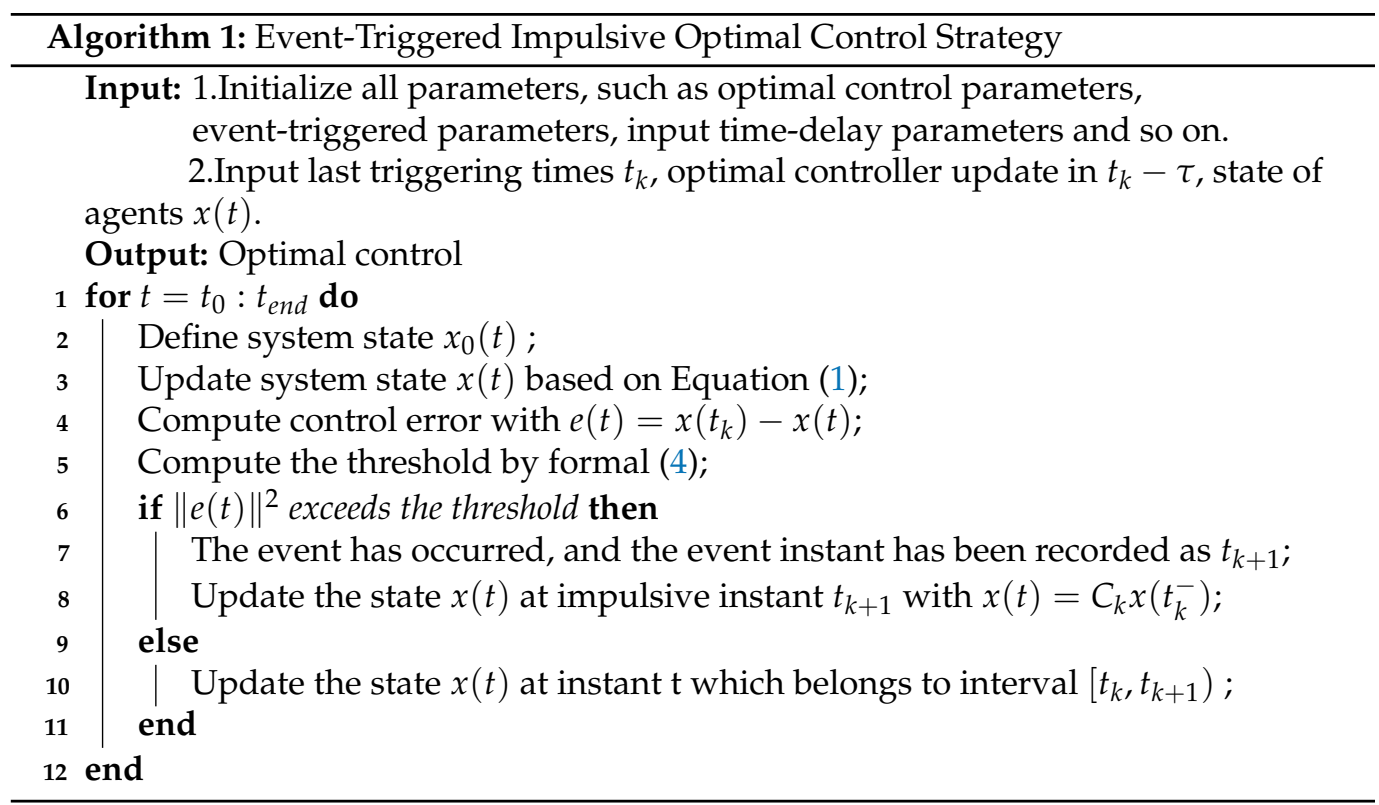




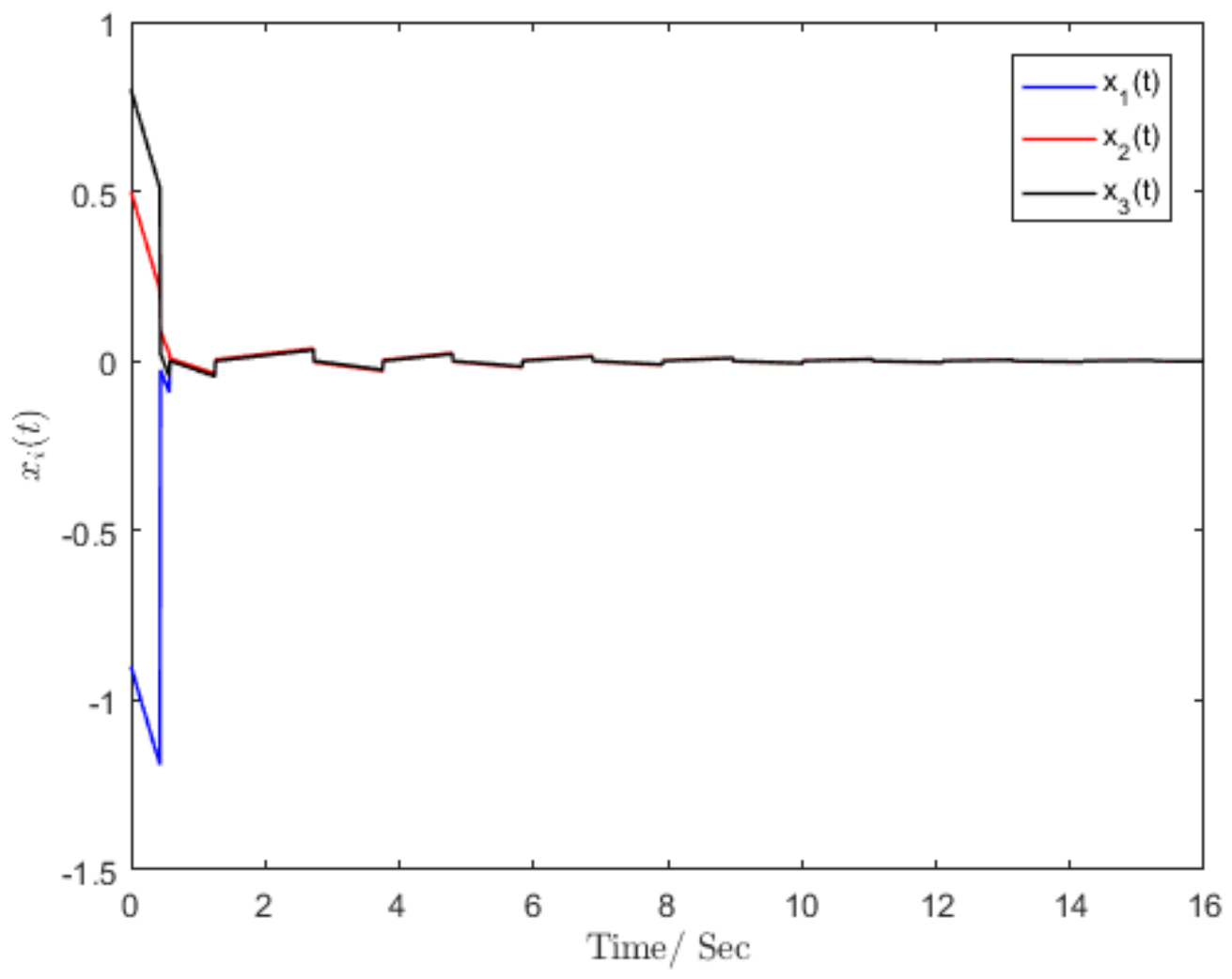

Figure 1. State of the system with $\tau=0.1$.

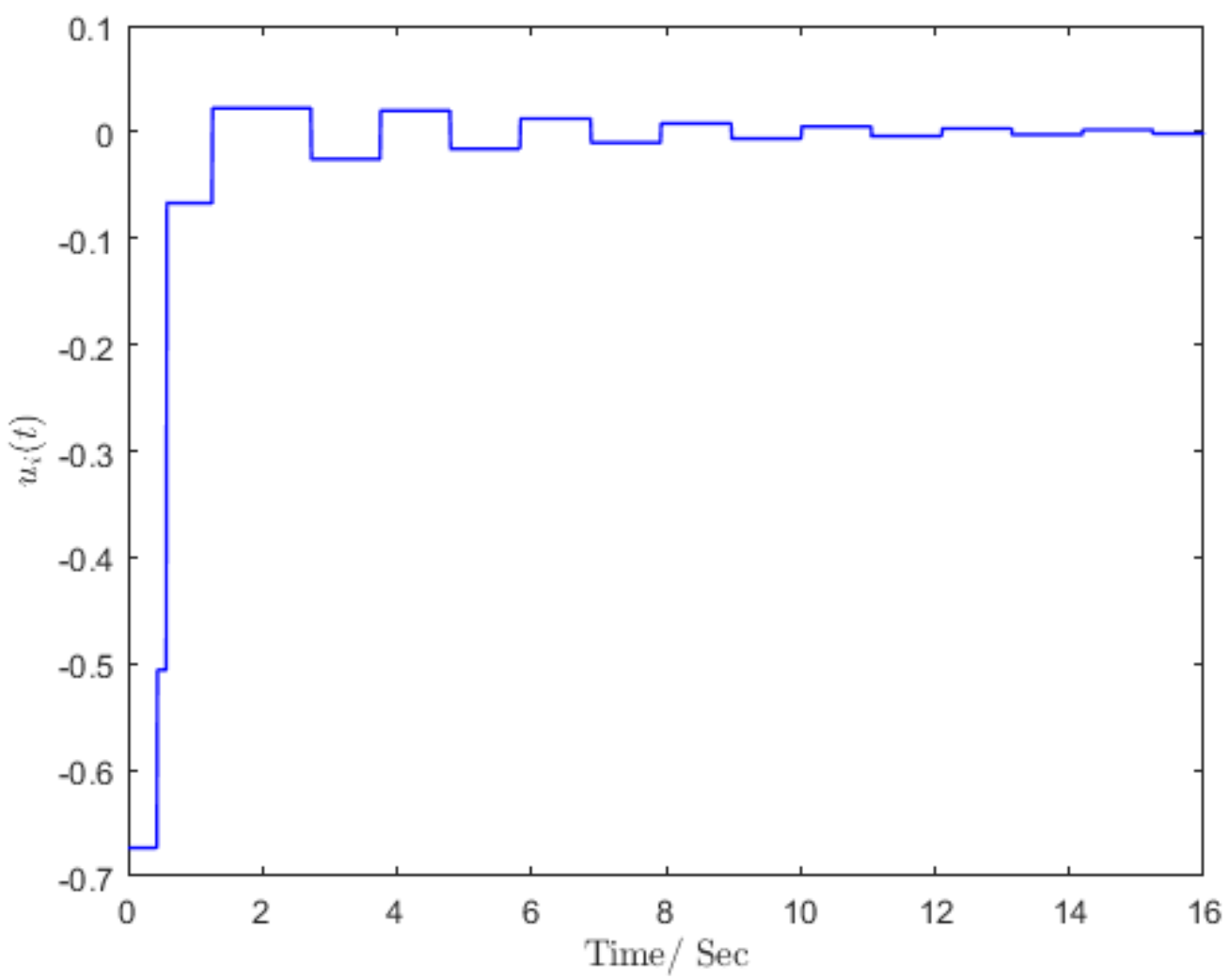

Figure 2. Controller with event-triggered impulse and $\tau=0.1$. 


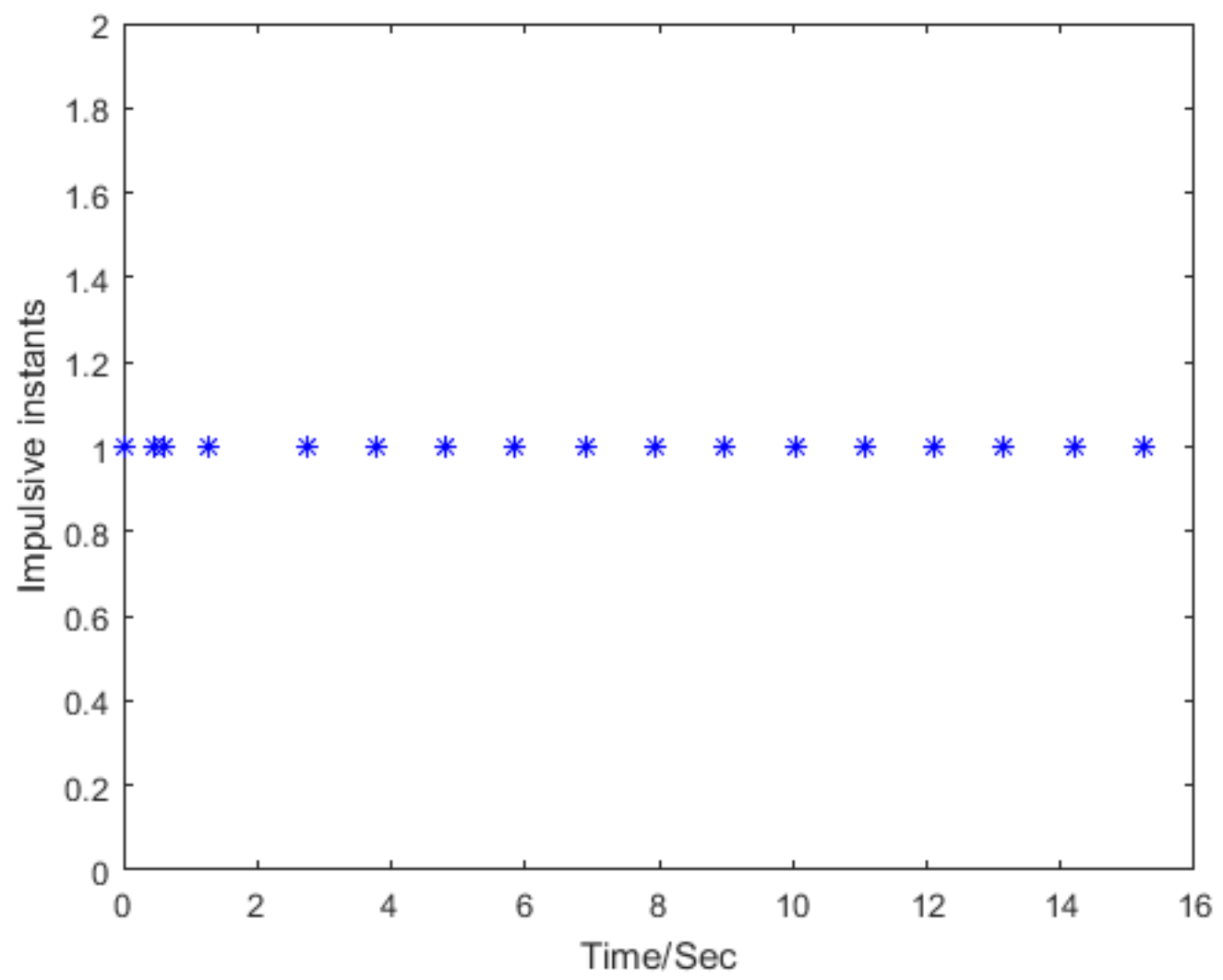

Figure 3. Impulsive instants with $\tau=0.1$.

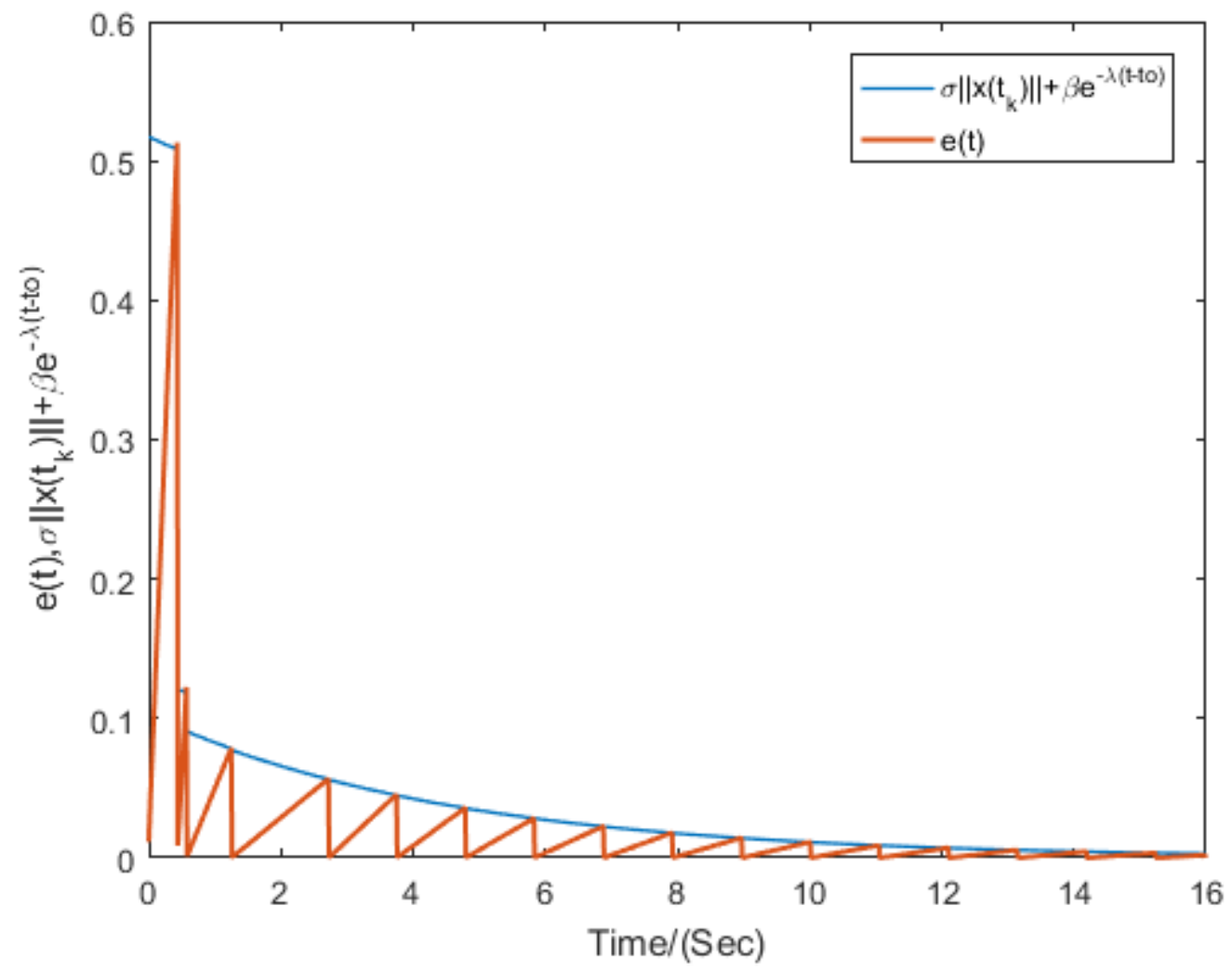

Figure 4. $\|e(t)\|$ and $\sigma\left\|x\left(t_{k}\right)\right\|+\beta e^{-\lambda\left(t-t_{0}\right)}$ with $\tau=0.1$. 


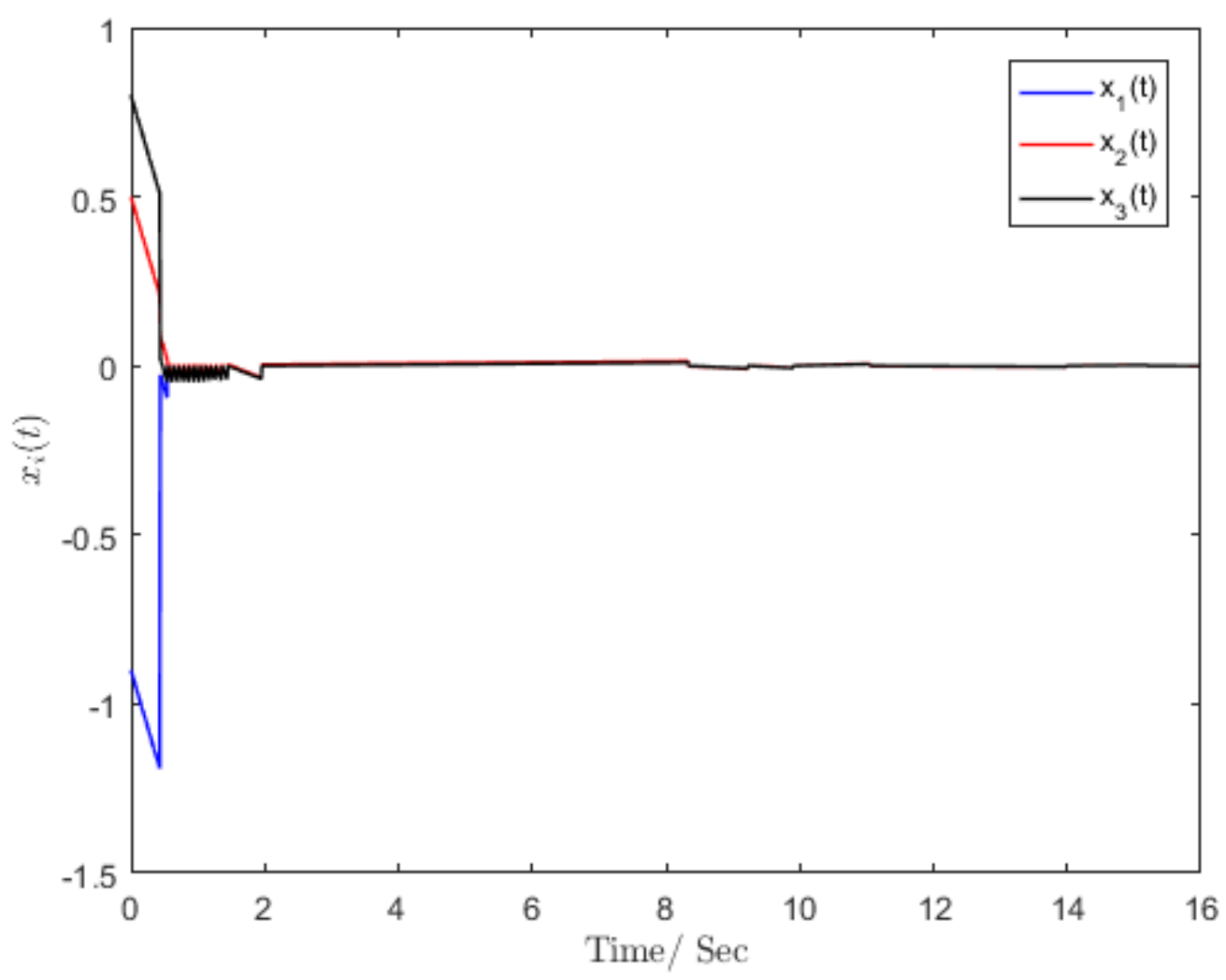

Figure 5. State of the system with $\tau=1$.

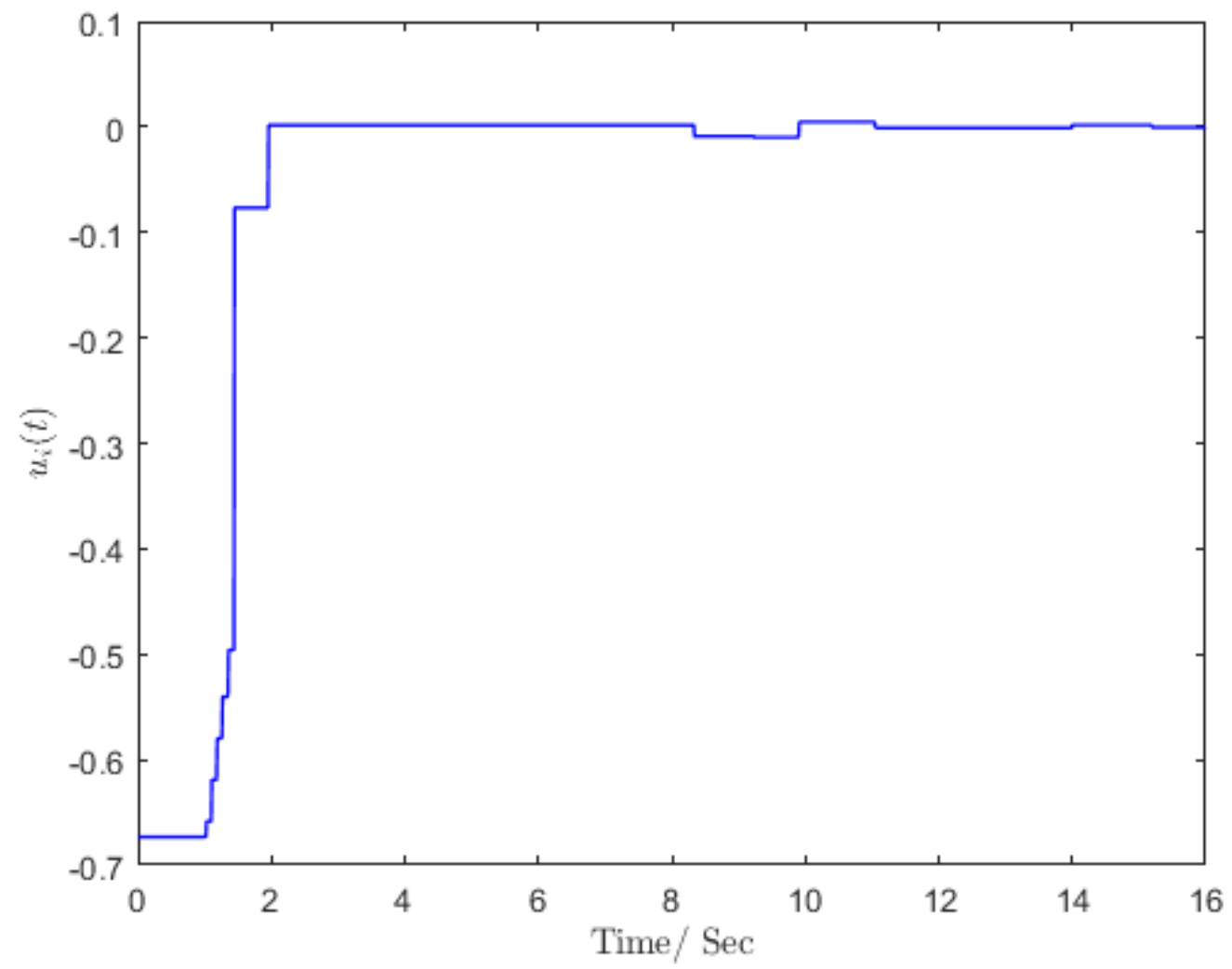

Figure 6. Controller with event-triggered impulse and $\tau=1$. 


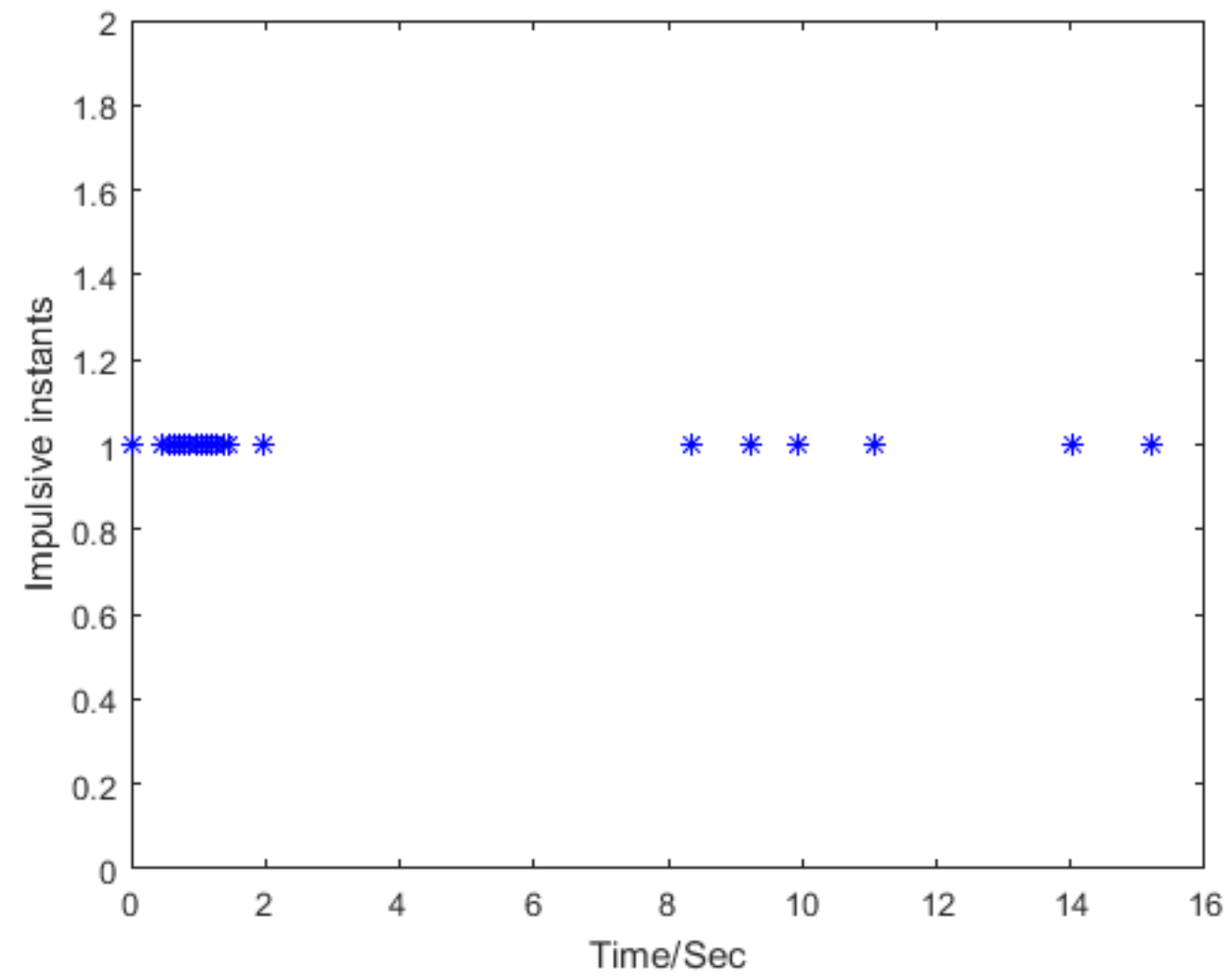

Figure 7. Impulsive instants with $\tau=1$.

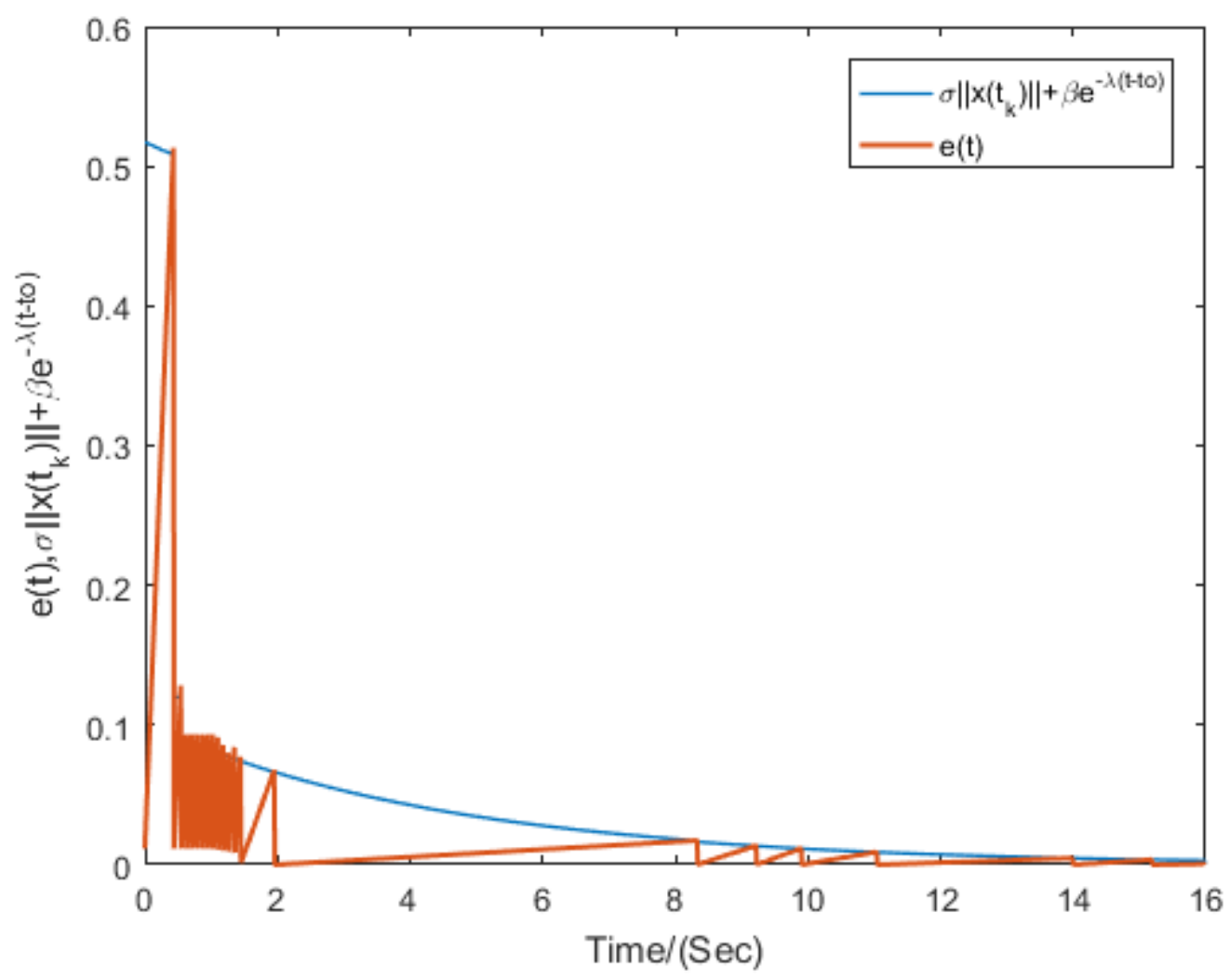

Figure 8. $\|e(t)\|$ and $\sigma\left\|x\left(t_{k}\right)\right\|+\beta e^{-\lambda\left(t-t_{0}\right)}$ with $\tau=1$.

Remark 2. From Algorithm 1, one can see that the impulsive control is an important factor which influences the convergence of the systems. Figure 9 shows that the event-triggered optimal control 
cannot be achieved without impulsive control, which implied that the impulsive control plays key role of the stability.

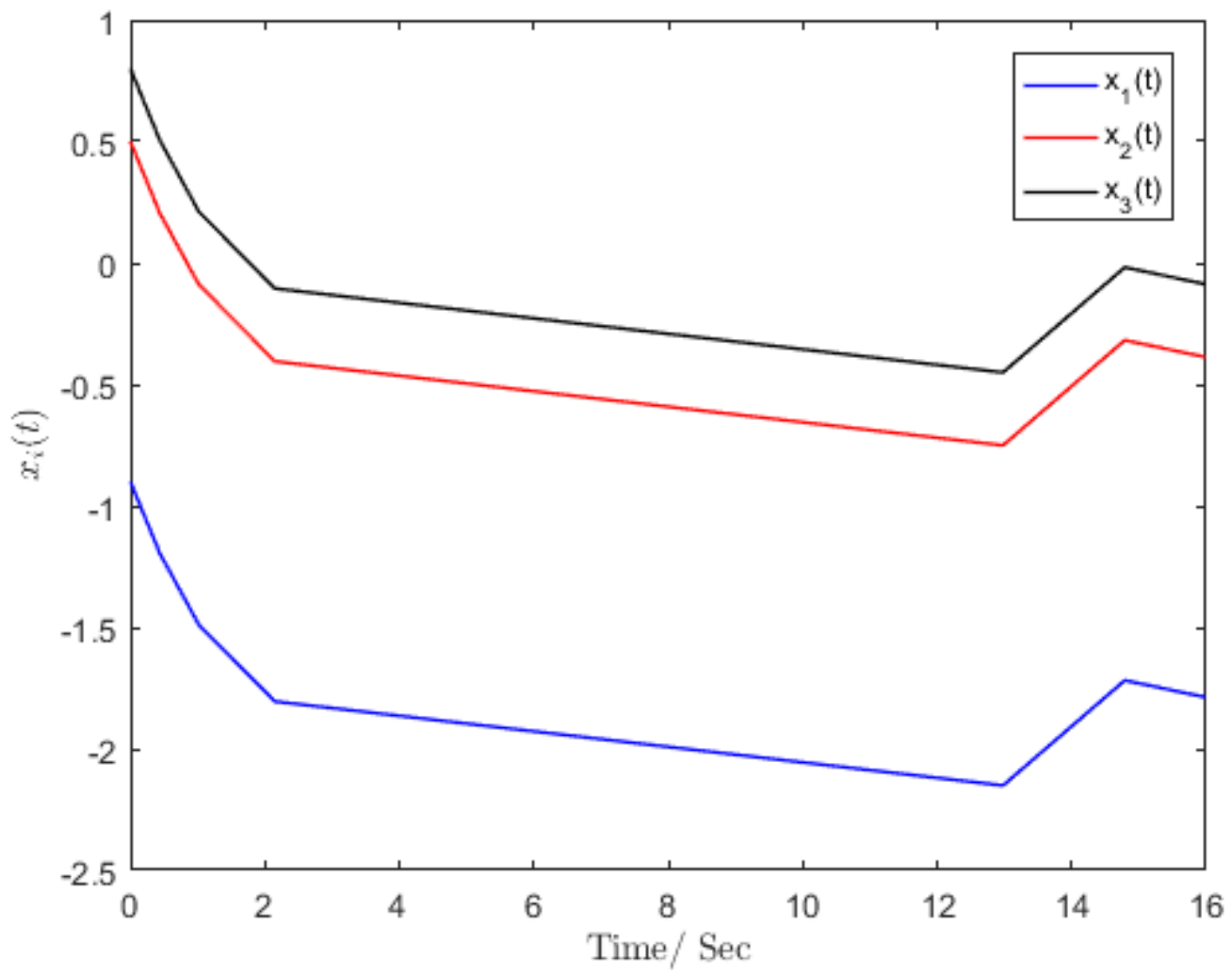

Figure 9. State of the system without impulse.

\section{Conclusions}

Based on optimal control theory and the stability theory of functional differential equations with impulse, the event-triggered impulsive optimal control for linear continuoustime dynamic systems with input time-delay is investigated in this paper. Considering the influence of input time-delay and impulsive jump, the performance index and impulsive control protocol were designed. Some sufficient conditions are given for guaranteeing the exponential stability with the optimal controller. The event-triggered impulsive optimal control problem for switching systems will be discussed in future.

Author Contributions: Writing—original draft preparation, visualization, Q.B.; supervision, funding acquisition, W.Z.; conceptualization, methodology, writing-review and editing, Q.B. and W.Z. All authors have read and agreed to the published version of the manuscript.

Funding: This research was funded by National Natural Science Foundation of China under Grant No.61673080, 61876200, the Science and Technology Research Program of Chongqing Municipal Education Commission under Grant KJZD-K202000601,KJZD-M202100602, Natural Science Foundation of Chongqing under Grant cstc2019jcyjmsxmX0102.

Acknowledgments: The authors would like to thank the Associate Editor and reviewers for their insightful comments and suggestions based on which the presentation of this paper has been greatly improved.

Conflicts of Interest: The authors declare no conflict of interest. 


\section{References}

1. Kaserer, D.; Gattringer H.; Mueller, A. Time optimal motion planning and admittance control for cooperative grasping. IEEE Robot. Autom. Lett. 2020, 99, 2216-2223. [CrossRef]

2. Chen, B.; Li, X.; Evangelou, S.A.; Lot, R. Joint propulsion and cooling energy management of hybrid electric vehicles by optimal control. IEEE Trans. Veh. Technol. 2020, 5, 4894-4906. [CrossRef]

3. Hu, Q.; Tan, X. Dynamic near-optimal control allocation for spacecraft attitude control using a hybrid configuration of actuators IEEE Trans. Aerosp. Electron. Syst. 2020, 56, 1430-1443. [CrossRef]

4. Gonzalez-Arriba, D.; Soler, M.; Sanjurjo-Rivo, M.; Kamgarpour, M.; Simarro, J. Robust aircraft trajectory planning under uncertain convective environments with optimal control and rapidly developing thunderstorms. Aerosp. Sci. Technol. 2019, 89, 445-459. [CrossRef]

5. Pan, H.J.; Ward, J.D. Dimensionless framework for seed recipe design and optimal control of batch crystallization. Ind. Eng. Chem. Res. 2021, 60, 3013-3026. [CrossRef]

6. Rueda-Escobedo, J.G.; Fridman, E.; Schiffer, J. Data-driven control for linear discrete-time delay systems. arXiv 2020, arXiv:2010.02657.

7. Hu, J.W.; Zhan, X.S.; Wu, J.; Yan, H.C. Optimal tracking performance of networked control systems with time-delay and encoding-decoding constraints. Int. J. Control. Autom. Syst. 2020, 18, 1012-1022. [CrossRef]

8. Gao, W.A.; Bl, A.; Shan, X.B. Integral reinforcement learning-based optimal output feedback control for linear continuous-time systems with input delay. Neurocomputing 2021, 460, 31-38.

9. Basin, M.; Rodriguez-Gonzalez, J. Optimal control for linear systems with time delay in control input. IEEE Trans. Autom. Control. 2004, 341, 267-278. [CrossRef]

10. Basin, M.; Rodriguez-Gonzalez, J. Optimal control for linear systems with multiple time delay in control input. IEEE Trans. Autom. Control. 2006, 51, 91-97. [CrossRef]

11. Meng, W.; Shi, J. A global maximum principle for stochastic optimal control problems with delay and applications. Syst. Control. Lett. 2021, 150, 104909. [CrossRef]

12. Ma, X.; Qi, Q.; Li, X.; Zhang, H. Optimal control and stablilization for linear continuous-time mean-field systems with delay arXiv 2020, arXiv:2010.08069v1.

13. Zhou, Y.S.; Wang, Z.H. Optimal feedback control for linear systems with input delays revisited. J. Optim. Theory Appl. 2014, 163, 989-1017. [CrossRef]

14. Pei, Y.; Chen, M.; Liang, X.; Li, C.; Zhu, M. Optimizing pulse timings and amounts of biological interventions for a pest regulation model. Nonlinear Anal. Hybrid Syst. 2018, 27, 353-365. [CrossRef]

15. Pang, L.; Xu, L. Impulsive control on a non-autonomous dispersal almost periodic competition system. Int. J. Comput. Sci. Math 2019, 10, 95-104. [CrossRef]

16. Abbasi, Z.; Zamani, I.; Mehra, A.; Shafieirad, M.; Ibeas, A. Optimal control design of impulsive SQEIAR epidemic models with application to COVID-19. Chaos Solitons Fractals 2020, 139, 110054. [CrossRef]

17. Mohammadi, M.S.; Naghash, A. Robust optimization of impulsive orbit transfers under actuation uncertainties. Aerosp. Sci. Technol. 2019, 85, 246-258. [CrossRef]

18. Liu, Z.W.; Wen, G.; Yu, X.; Guan, Z.H.; Huang, T. Delayed impulsive control for consensus of multiagent systems with switching communication graphs. IEEE Trans. Cybern. 2020, 50, 3045-3055. [CrossRef] [PubMed]

19. Yaghoubi, Z.; Talebi, H.A. Cluster consensus of fractional-order non-linear multi-agent systems with switching topology and time-delays via impulsive control. Int. J. Syst. Sci. 2020, 52, 1685-1698. [CrossRef]

20. Liu, X.; Chen, D.; Liu, Z.; Wang, Y.W. Distributed leaderless impulsive consensus of non-linear multi-agent systems with input saturation. Nonlinear Anal. Hybrid Syst. 2020, 36, 100855. [CrossRef]

21. Aarzén, K.E. A simple event-based PID controller. In Proceedings of the 14th World Congress of IFAC, Beijing, China, 5-9 July 1999.

22. Åström, K.J.; Bernhardsson, B.M. Comparison of Riemann and Lebesgue sampling for first order stochastic systems. In Proceedings of the 41st IEEE Conference on Decision and Control, Las Vegas, NV, USA, 10-13 December 2002.

23. Han, X.; Zhao, X.; Sun, T.; Wu, Y.; Zong, G. Event-triggered optimal control for discrete-time switched nonlinear systems with constrained control input. IEEE Trans. Syst. Man Cybern. Syst. 2020, 51, 7850-7859. [CrossRef]

24. Zhu, W.; Wang, D. Leader-following consensus of multi-agent systems via event-based impulsive control. Meas. Control.-Lond.Inst. Meas. Control. 2019, 52, 91-99. [CrossRef]

25. Li, X.; Yang, X.; Cao, J. Event-triggered impulsive control for nonlinear delay systems. Automatica 2019, 117, 108981. [CrossRef]

26. Peng, D.; Li, X. Leader-following synchronization of complex dynamic networks via event-triggered impulsive control. Neurocomputing 2019, 412, 1-10. [CrossRef]

27. Dan, L.A.; Dan, Y. Exponential synchronization of memristive delayed neural networks via event-based impulsive control method-Sciencedirect. J. Frankl. Inst. 2020, 357, 4437-4457.

28. Peng, D.; Li, X.; Rakkiyappan, R.; Ding, Y. Stabilization of stochastic delayed systems: Event-triggered impulsive control. Appl. Math. Comput. 2021, 401, 126054. [CrossRef]

29. Wei, L.; Chen, W.H.; Luo, S. Stabilization of discrete-time switched linear systems with time-varying delays via nearly-periodic impulsive control. J. Frankl. Inst. 2019, 356, 8996-9022. [CrossRef] 
30. Luo, S.; Deng, F.; Chen, W.H. Stability and stabilization of linear impulsive systems with large impulse-delays: A stabilizing delay perspective. Automatica 2021, 127, 109533. [CrossRef]

31. Sobiesiak, L.A.; Damaren, C.J. Linear quadratic optimal control for systems with continuous and impulsive inputs. In Proceedings of the 53th IEEE Conference on Decision and Control, Los Angeles, CA, USA, 15-17 December 2014.

32. Zhu, W.; Wang, D.; Liu, L.; Feng, G. Event-based impulsive control of continuous-time dynamic systems and its application to synchronization of memristive neural networks. IEEE Trans. Neural Networks Learn. Syst. 2018, 29, 3599-3609.

33. Zhu, W.; Yan C. Consensus analysis of second-order agents with active leader and time delay via impulsive control. In Proceedings of the 30th Chinese Control Conference, Yantai, China, 22-24 July 2011.

34. Zhu, W.; Jiang, Z.P. Event-based leader-following consensus of multi-agent systems with input time delay. IEEE Trans. Autom. Control. 2015, 60, 1362-1367. [CrossRef] 